\title{
A simple X-ray position detection system based on a MHSP
}

\author{
H. Natal da Luz ${ }^{\mathrm{a}, \mathrm{b}}$, J.F.C.A. Veloso ${ }^{\mathrm{a}, *}$, J.M.F. dos Santos ${ }^{\mathrm{b}}$, J.A. Mir ${ }^{\mathrm{c}}$ \\ ${ }^{a}$ Physics Department, University of Aveiro, Campus Universitário de Santiago, 3810-193 Aveiro, Portugal \\ ${ }^{\mathrm{b}}$ Physics Department, University of Coimbra, 3004-516 Coimbra, Portugal \\ ${ }^{\mathrm{c}}$ Instrumentation Department, CCLRC Rutherford Appleton Laboratory, Chilton, Didcot, Oxon OX11 0QX, UK
}

Available online 5 July 2007

\begin{abstract}
A simple system for position detection based on a Micro-Hole and Strip Plate (MHSP) using a resistive charge division method was implemented. A thin resistive layer of about $100 \Omega$ between each strip (top surface) and between each anode strip (bottom surface) allows us to obtain the actual position in both orthogonal directions. The readout electronics uses only two preamplifiers, a sum and a divider circuit to calculate the interaction point, in each dimension. For a detector filled with $1 \mathrm{~atm}$ of pure xenon, position resolutions of less than $300 \mu \mathrm{m}$ (FWHM) were achieved using $5.9 \mathrm{keV}$ X-rays, for a signal-to-noise ratio (SNR) of 30. For these conditions, a detector gain of about $10^{4}$ and an energy resolution of about $15 \%$ were obtained.

(C) 2007 Elsevier B.V. All rights reserved.
\end{abstract}

PACS: 29.40.Gx; 29.40.Cs; 87.50.Gi; 87.59.-e

Keywords: Micro-Hole and Strip Plate; Gas Electron Multiplier; 2D imaging; Resistive charge division; Analogue divider; X-ray detectors

\section{Introduction}

$\mathrm{X}$-ray imaging is a very powerful tool, used in several areas of knowledge. Being first used in medicine for diagnosis, it has quickly been developed to fields such as fluorescence studies, crystallography, biology and even neutron physics. For experiments that do not require very high energy and position resolutions, gas detectors may have advantages over semiconductor detectors, in terms of cost and maintenance. The recent development of gaseous detectors using microstructures provided new perspectives for X-ray detection and imaging.

A new microstructure has been studied in the last few years. The Micro-Hole and Strip Plate (MHSP) is a combination of a Micro-Strip Gas Chamber (MSGC) and a Gas Electron Multiplier (GEM) in a single double sided structure. Like the GEM, the MHSP is fabricated with printed circuit board (PCB) technology from a $50-\mu \mathrm{m}$ Kapton ${ }^{\mathrm{TM}}$ film, metallised with 5 - $\mu \mathrm{m}$-thick copper layers on both sides. The working principle of the MHSP has

\footnotetext{
${ }^{*}$ Corresponding author.

E-mail address: joao.veloso@ua.pt (J.F.C.A. Veloso).
}

been already explained in Ref. [1], as well as the initial idea for imaging with this device. This hybrid detector has already revealed very competitive characteristics in terms of gain, energy resolution [2], count rate and stability. Since the MHSP has an intrinsic 2D imaging capability, by separating the anodes on the MSGC side and structuring the GEM side also in separated electrodes, the possibility of imaging with such a simple and low-cost device is to be explored. The aim of this work is to develop an X-ray imaging system based on a MHSP specially developed for resistive charge division readout, to infer its performance in applications such as X-ray imaging. The electronics has also been developed by us, using a commercial analogue divider.

\section{Experimental set-up and methods}

A stainless steel vessel was built to contain the MHSP structure in a 1-bar xenon atmosphere. The absorption region was produced by placing the MHSP at a distance of $5 \mathrm{~mm}$ from the aluminised Mylar ${ }^{\mathrm{TM}}$ window. The top side copper layer of the MHSP is patterned with $60-\mu \mathrm{m}$ diameter double-conic holes, which cross the Kapton ${ }^{\mathrm{TM}}$ 
foil. The holes are separated by $140 \mu \mathrm{m}$ on a hexagonal pattern. On the bottom side, the holes are aligned in the middle of the cathodes of a micro-strip-like pattern with $100-\mu \mathrm{m}$ wide cathodes and about $20-\mu \mathrm{m}$ anodes, with a pitch of $200 \mu \mathrm{m}$.

For this work, the top side of the structure has been structured in separated strip-like electrodes perpendicular to the MSGC strips. For 2D-position readout both the upper strips and the anode strips were connected by resistive lines as shown in Fig. 1. The typical values of the resistance between both edges of the resistive lines are between 15 and $25 \mathrm{k} \Omega$, leading to a resistance between anodes of about $150 \Omega$. In order to obtain images, only four charge preamplifiers are needed - two for each dimension. After being converted to voltage signals by the charge preamplifiers, the signals are shaped by two amplifiers with $0.5-\mu$ s shaping time. One of the signals and the sum of both are stretched and an analogue divider (AD754) provides a signal amplitude proportional to $X_{\mathrm{L}} /\left(X_{\mathrm{L}}+X_{\mathrm{R}}\right)$, where $X_{\mathrm{L}}$ and $X_{\mathrm{R}}$ are the amplitudes of the signals coming from the left and the right edges of the resistive line, for the $X$ dimension. No energy window discrimination was applied.

The signals collected from the GEM side of the MHSP are induced by the charge on the anodes on the MSGC side. Their amplitude is about $35 \%$ of that on the anodes [2]. Since the position resolution is dependent on the signalto-noise ratio (SNR) of the system [3], the position resolution is expected to be lower for the dimension obtained for the top side. In this work the results for the signals collected from the anodes and from the top side electrodes will be presented independently.

\section{Experimental results and discussion}

The detector window is attached to a collimator, which consists of a $5 \times 5$ matrix of 2 -mm holes with a distance of $6 \mathrm{~mm}$ between their centres. To obtain the resulting position distributions, a ${ }^{55} \mathrm{Fe} \mathrm{X}$-ray source (Mn K lines) was used to irradiate one row of the collimator holes, while the other holes were kept covered. The distance between the X-ray source and the window was about $30 \mathrm{~cm}$. Since the position resolution is highly dependent on the SNR, it is of interest that the detector works with the highest gain possible. However, for higher voltages, the number of microdischarges starts to increase, jeopardising the integrity of the microstructure. This means that the highest safe polarisation voltages were used, i.e., inexistence of electrical discharges. A maximum gain of $10^{4}$ corresponding to an SNR of 30 was achieved.

Fig. 2a shows the position distribution corresponding to the projections of four holes in one dimension obtained with this system (the fifth hole was not seen by the MHSP, because it was not centred with the detector window), with the signals read from the anodes on the micro-strip side. The shaded circles above the peaks represent the collimator holes scaled to the position distribution. The shape of the peaks is the expected stretched half circle, indicating that the resolution is much better than the $2-\mathrm{mm}$ diameter of the holes. The second hole from the left edge was partially covered by the glue used to attach the collimator to the window, as it can be seen in this 1D projection. The position linearity of the system is also shown in the graphic. The standard deviation from linearity of the data points

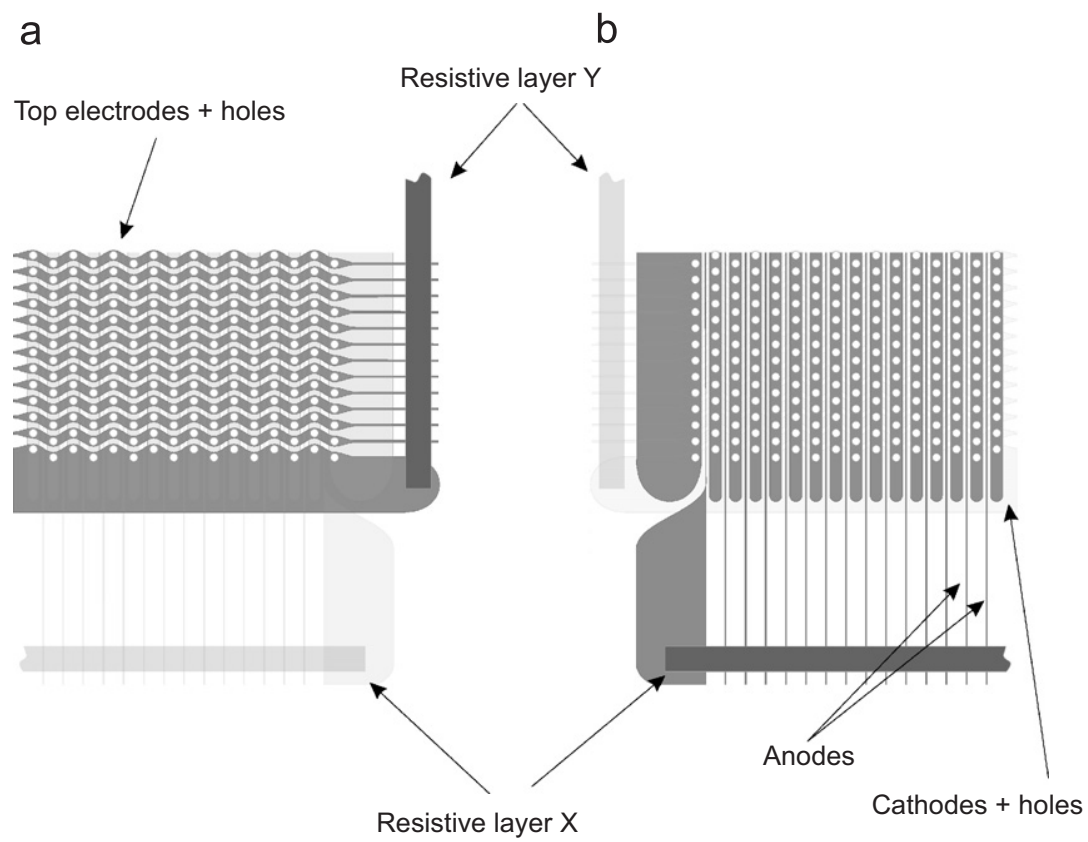

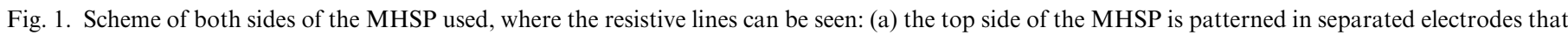

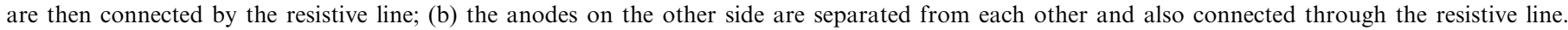
The resistive lines are perpendicular to each other. 
a

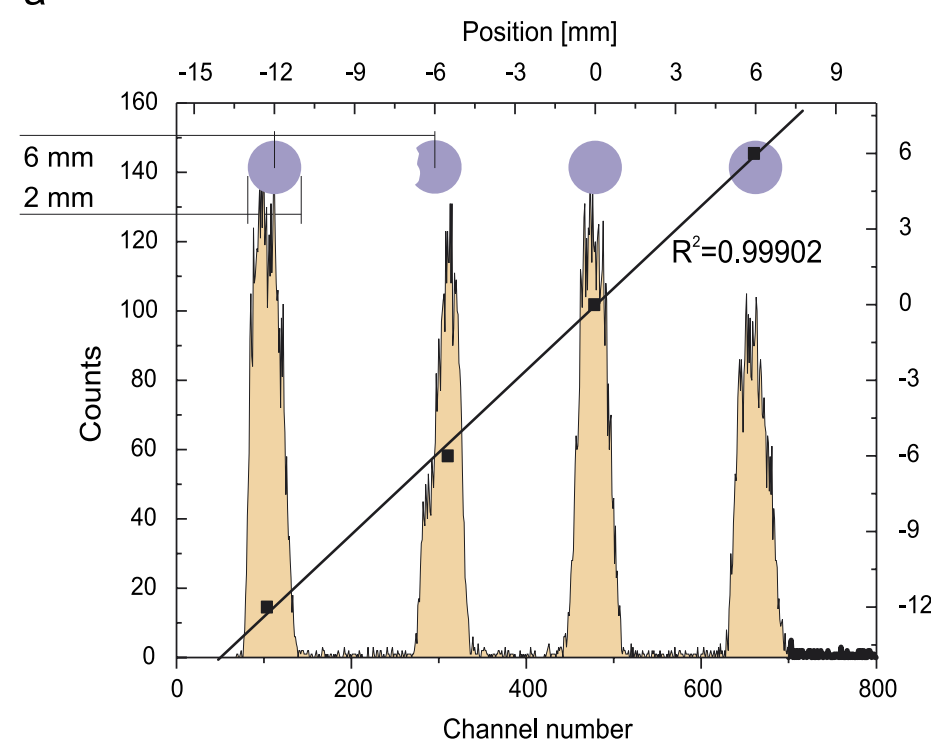

b

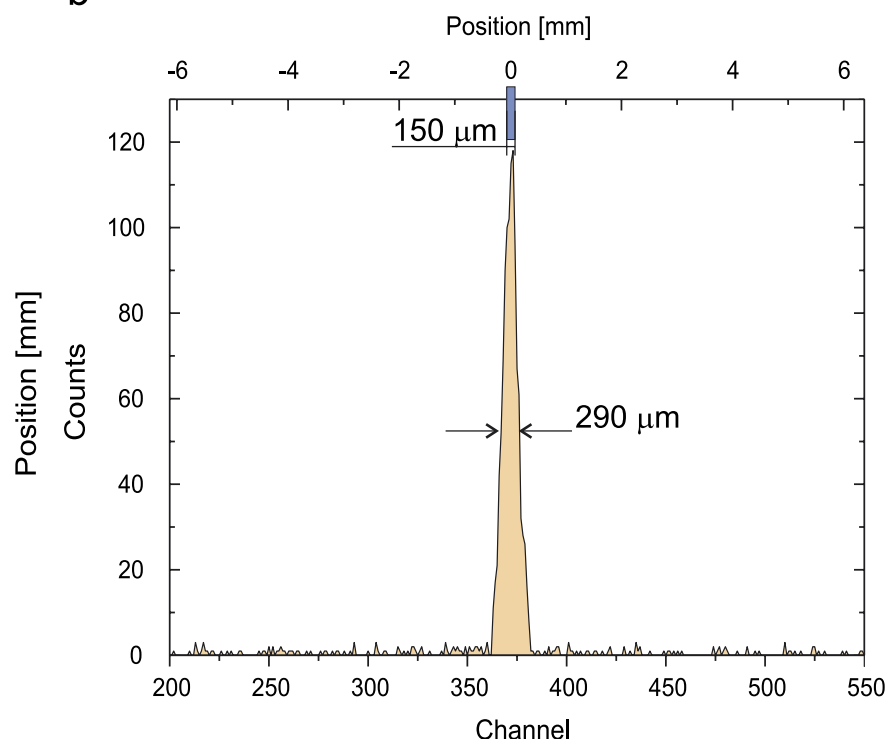

Fig. 2. (a) The $1 \mathrm{D}$ projection of four holes of the collimator (scaled with the position distribution above the peaks), with the signals collected from the anodes of the MHSP, showing the position linearity. (b) Position distribution obtained for a $150-\mu \mathrm{m}$ slit. FWHM $=290 \mu \mathrm{m}$.

a

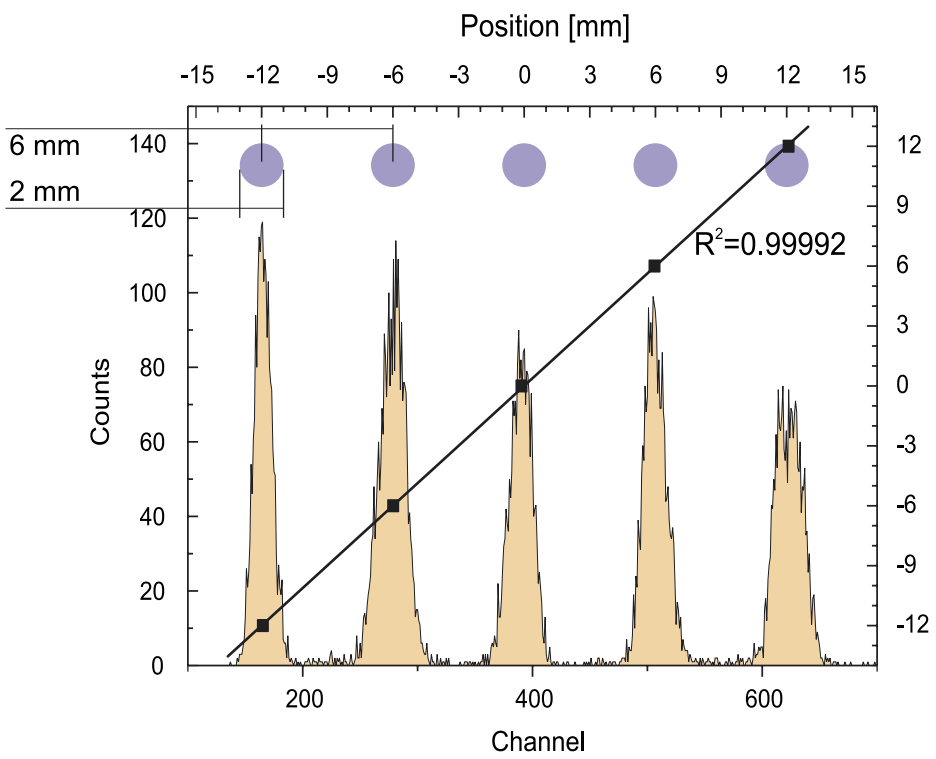

b

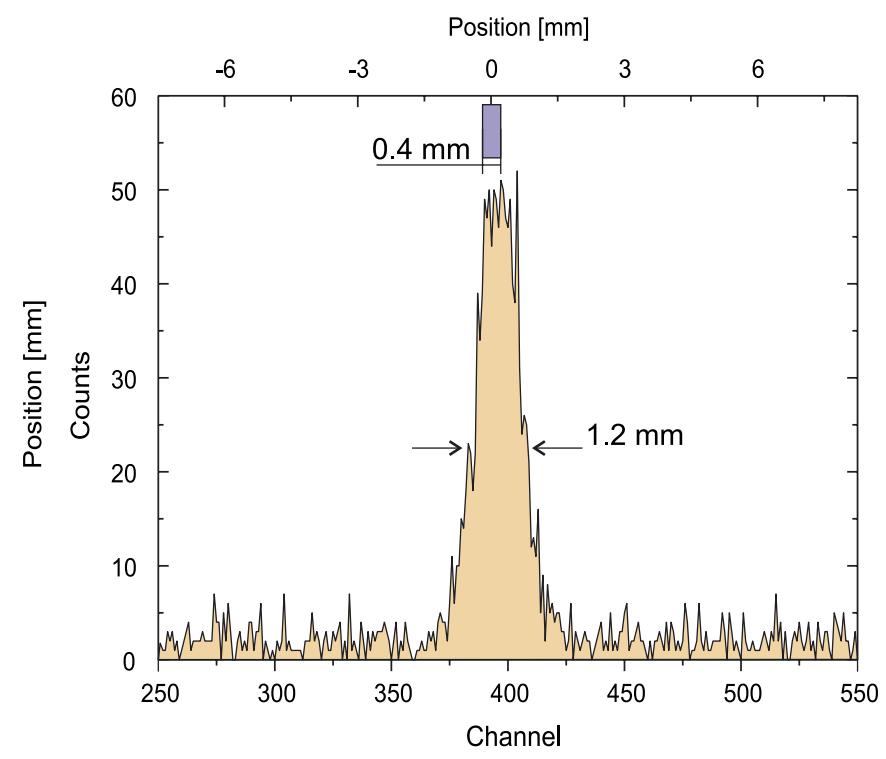

Fig. 3. (a) The 1D projection of five holes of the collimator (scaled with the position distribution above the peaks), with the signals collected from the top electrodes of the MHSP, showing the position linearity. (b) Position distribution obtained for a 0.4-mm slit. FWHM $=1.2 \mathrm{~mm}$.

in this graph corresponds to around $66 \mu \mathrm{m}$. In Fig. 2b, a $150-\mu \mathrm{m}$ slit was used on the middle hole. The peak is a convolution of a Gaussian distribution associated to the intrinsic resolution of the system and a rectangular projection of the slit. Considering it purely Gaussian, the FWHM is found to be $290 \mu \mathrm{m}$. The real position resolution is somewhat better, if the deviation of this peak from a Gaussian is taken into account [4].

Preliminary studies, for the second coordinate measured on top electrodes of the MHSP, were performed using the same methods. As mentioned, the SNR is lower in this case (about 10), which predicts a worse position resolution for this dimension. It can be seen that the shape of the peaks is more close to a Gaussian shape, which indicates that the contribution of the position resolution becomes more important. However, it can be seen that the position linearity is still very good. In Fig. 3b, a $0.4-\mathrm{mm}$ slit was used, and a FWHM of $1.2 \mathrm{~mm}$ was achieved. In this case, a standard deviation from linearity was found to be $75 \mu \mathrm{m}$.

Finally, in Fig. 4, an aluminium collimator was built to fit in one of the 2-mm holes, containing a row of three $0.5-\mathrm{mm}$ diameter holes, and a distance of $0.75 \mathrm{~mm}$ between 


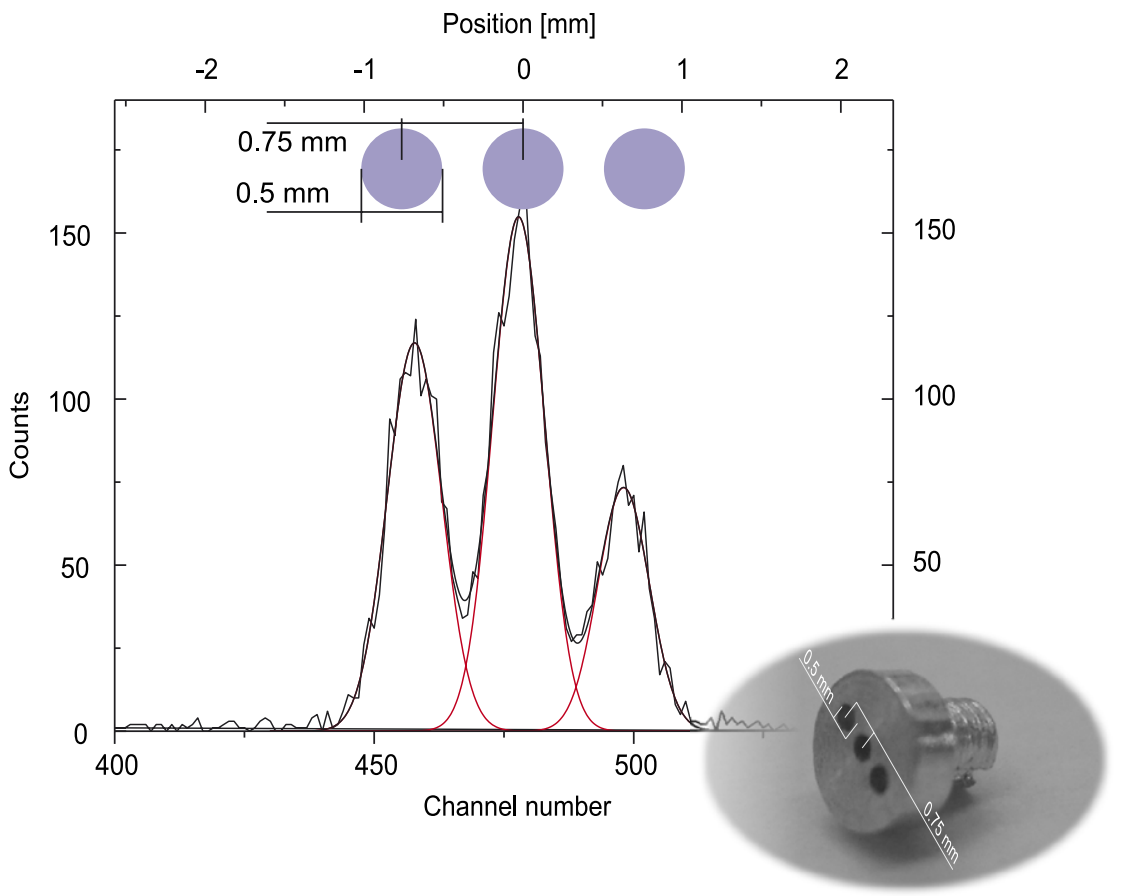

Fig. 4. The 1D projection of the aluminium collimator shown. The three peaks corresponding to each of the 0.5 -mm holes are perfectly distinguished.

their centres. The three expected peaks are completely distinguished when collecting the signals from the microstrip side.

\section{Conclusions}

The results presented in this work have shown that the MHSP is a very promising solution for X-ray imaging at a low price. It is able to provide position resolutions that are perfectly suited for many applications with X-rays. The materials used are standard, and the principle of operation is very simple, which makes this device very cost effective. Furthermore, the processing electronics needed to obtain images is much simplified due to the resistive charge division.

Position resolutions of less than $300 \mu \mathrm{m}$ were achieved for the dimension taken from the anode strips, while for the dimension read from the upper strips a position resolution of $1.2 \mathrm{~mm}$ was reached.

Due to the strong dependence of the position resolution on the MHSP gain, better quality MHSPs, with higher gains, will allow position resolution improvement. Optimisation of the resistive layers will also allow higher count rate capability, enough to make this device even more efficient for some medical applications. The position resolutions achieved are nevertheless already compatible with the use of this detector in neutron spallation sources.

\section{Acknowledgements}

H. Natal da Luz is supported by Fundação para a Ciência e a Tecnologia through Doctoral Grant SFRH/ $\mathrm{BD} / 16342 / 2004$. This work was supported in part by Project POCI/FP/63414/2005 through FEDER and FCT (Lisbon) programs.

\section{References}

[1] J.F.C.A. Veloso, J.M.F. dos Santos, C.A.N. Conde, Rev. Sci. Instr. 71 (6) (2000) 2371

[2] H. Natal da Luz, J.F.C.A. Veloso, F. Amaro, L.F. Requicha Ferreira, J.M.F. dos Santos, A. Breskin, R. Chechik, Nucl. Instr. and Meth. A $552(2005) 259$.

[3] J.P. Cussonneau, M. Labalme, P. Lautridou, L. Luquin, V. Metivier, A. Rahmani, T. Reposeur, Nucl. Instr. and Meth. A 492 (2002) 26.

[4] H. Klein, H. Brede, B.R.L. Siebert, Nucl. Instr. and Meth. 193 (1982) 635. 